\title{
La percepción de la pobreza bajo el ángulo de la teoría de la vinculación: naturalización, culpabilización y victimización ${ }^{1}$
}

Serge Paugam*

\begin{abstract}
Resumen
La naturalización, la culpabilización y la victimización constituyen tres formas difusas de percepción de la pobreza e inevitablemente presentes en todas las sociedades. Este artículo estudia, más allá de los factores de variación generalmente tomados en cuenta, lo que se refiere más específicamente a los modos de regulación de los vínculos sociales que se pueden analizar a partir de una tipología de configuraciones de vinculación.
\end{abstract}

Palabras clave: Pobreza - teoría de la vinculación - lazos sociales.

\begin{abstract}
Naturalization, criminalization and victimization are three forms of perception of poverty inevitably present in all societies. Beyond the factors of change generally taken into account, this article inquires more specifically into the modes of regulation of social bonds which can be analyzed using a typology of attachment configurations as a starting point.
\end{abstract}

Keywords: Poverty - theory of linkage - social bonds.

1 Traducción realizada por Clemencia González Tugas y Claudia Jordana Contreras (ERIS/CMH).

* Director de Estudios, L'École des Hautes Études en Sciences Sociales (EHESS), Francia. Director de Investigación, Centre National de la Recherche Scientifique (CNRS), Equipe de Recherche sur les Inegalités Sociales (ERIS)/Centre Maurice Halbwachs (CMH), Francia. Correo electrónico: serge.paugam@ehess.fr 


\section{INTRODUCCIÓN ${ }^{1}$}

En ciertas sociedades la pobreza está tan extendida que aparece como la condición ineludible de una fracción importante de la población. Esta se asocia en las representaciones sociales a un orden social inmutable donde los pobres son a la vez inferiorizados e integrados. Estos últimos tienen el sentimiento de pertenecer a lo que podemos Ilamar una "comunidad de destino": el sistema de desigualdades es tan rígido que los pobres saben que no tienen ninguna posibilidad de mejorar su suerte. La única solución para sobrevivir es la de contar con sus cercanos, sobre todo porque ninguna acción pública de gran alcance es llevada a cabo para ayudarlos. Esta comunidad, que ha pertenecido de generación en generación al último estrato de la sociedad, tiene una enorme probabilidad de ser percibida por los otros como la expresión de desigualdades de naturaleza biológica o cultural. Se trata, en otras palabras, de un proceso de naturalización de la pobreza. En contraste, en otras sociedades, es habitual considerar que la pobreza es la expresión de injusticias intolerables. La condición material y el sufrimiento de los pobres ponen en cuestión los principios fundadores y reguladores de la cohesión social y ciudadana; la pobreza debe ser combatida en nombre del interés colectivo de vivir en una sociedad de semejantes. En otros términos, los pobres son víctimas y es más bien la sociedad la que debe reformarse para que no lo sean más. La "naturalización" y la "victimización" de la pobreza pueden ser consideradas como dos polos extremos; entre ellos existiría una percepción intermedia que podríamos Ilamar "culpabilización" de los pobres. Algunas sociedades no se refieren a un orden social natural donde los pobres estarían llamados a ocupar funciones inferiores, pero tampoco adhieren plenamente a la imagen opuesta donde estos serían víctimas por las cuales habría que movilizarse colectivamente. Los pobres deben hacerse cargo ellos mismos de aprovechar las oportunidades que el sistema social puede procurarles; en caso de no lograrlo, ellos son legítimamente considerados como incapaces, irresponsables o perezosos.

Estos tres grandes tipos de percepción de la pobreza pueden estar presentes simultáneamente en cada sociedad, ya que las representaciones sociales no son totalmente homogéneas y son susceptibles de variar según la clase social, el género, la edad, la trayectoria biográfica, e incluso según la coyuntura o las fases del crecimiento económico y de la protección social $^{2}$. Sin embargo, pueden estar igualmente asociados a ciertos tipos de sociedad, ya que una u otra de estas tres percepciones puede ser dominante e imponerse como un rasgo antropológico e histórico de su desarrollo (Paugam, 2005). Es con ese ánimo que se plantea la problemática de este artículo. Creemos que es posible superar, aunque sin rechazar, la explicación de estas diferencias de percepción a partir de las variables socioeconómicas y coyunturales. Para ello consideraremos un análisis más estructural a partir de un marco teórico determinado, el de la vinculación, esto es, un sistema de entrecruzamiento normativo

Agradezco a Camila Giorgetti, Jules Naudet, Dominique Schnapper y a François-Xavier Schweyer; a los directores de este número; y a los relectores anónimos así como a los participantes de mi seminario de dirección de estudios en EHESS, por sus observaciones y sugerencias a este texto.

2 Leeremos particularmente a Geremek (1987), Piven y Cloward (1993), Van Oorschot y Halman (2000), Paugam y Selz (2005) y Paugam (2013). 
de los lazos sociales. Nos dedicaremos en un primer momento a presentar dicho marco. En un segundo momento nos dispondremos a someterlo a la verificación empírica a partir de trabajos comparativos Ilevados a cabo especialmente a nivel europeo, pero también a partir de trabajos más recientes o en desarrollo en terrenos no europeos.

\section{ANALIZAR LA TRAMA DE LA SOCIEDAD}

La hipótesis que planteamos es que la percepción de la pobreza, así como puede ser estudiada en una sociedad, constituye en cierta forma la expresión de una representación colectiva más global, que remite a los lazos que unen a los individuos entre ellos y a la sociedad en su conjunto. Esta hipótesis está notoriamente influida por la definición de la moral que elabora Durkheim en la conclusión de su libro La división del trabajo social: "Puede decirse que es moral todo lo que constituye fuente de solidaridad, todo lo que fuerza al hombre a contar con otro, a regular sus movimientos con arreglo a algo más que los impulsos de su egoísmo, y la moralidad es tanto más sólida cuanto más numerosos son sus lazos y más fuertes" (Durkheim, 2007a: 394). Según él, la sociedad es la condición necesaria de la moral. Dicho de otra manera, en esta perspectiva analítica, la percepción de la pobreza está en gran parte determinada por una moral colectiva, que no puede entenderse sino como el reflejo de los lazos sociales o de solidaridad.

¿Cuáles son esos lazos? La pregunta, como sabemos, anima a Durkheim desde su tesis acerca de la división del trabajo social, y la reencontramos en su estudio del suicidio (Durkheim, 2007b) y en sus lecciones impartidas en Burdeos durante la última década del siglo XIX, donde aparece de manera explícita la vinculación a los grupos como una de las fuentes esenciales de La educación moral (Durkheim, 2012). Distinguiremos cuatro tipos de lazos sociales: el lazo de filiación (en el sentido de las relaciones parentales), el lazo de participación electiva (en el sentido de las relaciones entre pares elegidos libremente), el lazo de participación orgánica (en el sentido de la solidaridad orgánica y de la integración profesional) y el lazo de ciudadanía (en el sentido de las relaciones de igualdad entre miembros de una misma comunidad política) (Paugam, 2013b). Cada lazo puede ser definido a partir de dos dimensiones: protección y reconocimiento. La protección se refiere al conjunto de soportes que el individuo puede movilizar frente a los avatares de la vida (recursos familiares, comunitarios, profesionales, sociales...), y el reconocimiento remite a las interacciones sociales que animan al individuo otorgándole la confirmación de su existencia y de su valoración por medio de la mirada del otro o de los otros. La expresión "contar con" resume bastante bien lo que el individuo puede esperar de su relación con los demás y con las instituciones en términos de protección, mientras que la expresión "contar para" expresa su expectativa, también vital, de reconocimiento.

Este marco analítico se aleja sustancialmente de los trabajos clásicos desarrollados por la sociología de redes respecto de la fuerza de los lazos débiles (Granovetter, 1973). De acuerdo con nuestra definición, la fuerza no se mide únicamente en una relación interpersonal, sino también en la vinculación al sistema social que hace o no posible un conjunto de relaciones interpersonales inscritas en esferas normativas distintas. El lazo, como 
lo entendemos, es un lazo en el sentido durkheimiano de la vinculación a la sociedad, lo que implica tomar en cuenta el sistema normativo que lo constituye, de acuerdo con la hipótesis de que los individuos se ven más o menos constreñidos a conformarse a este para integrarse. En el lazo de filiación, por ejemplo, se estudia la relación entre padres e hijos, pero remitiendo esa relación a las normas que la enmarcan en una sociedad determinada, en el entendido que la filiación puede asumir formas diferentes de una sociedad a otra. En el lazo de participación electiva, la intensidad de la relación entre individuos en el seno de comunidades organizadas, bajo el modo de agrupación por afinidad, será variable según las expectativas de esas comunidades, pero también según la amplitud de la incitación normativa que las sociedades ejerzan en sus miembros para que se involucren en ese tipo de estructuras, en nombre de valores compartidos y susceptibles de asegurar la cohesión social. En el lazo de participación orgánica, se estudia la relación entre agentes que participan en la vida profesional, considerando que esa relación varía si se trata de una sociedad salarial consolidada o de una sociedad salarial incompleta, de una sociedad salarial en crisis o una sociedad salarial en expansión. Por último, en el lazo de ciudadanía, lo que está en juego es la protección de los individuos en virtud de sus derechos civiles, políticos, económicos y sociales y al reconocimiento de su calidad de individuos soberanos ${ }^{3}$.

Esta tipología permite también entender cómo los lazos sociales están entrecruzados normativamente en cada sociedad y cómo a partir de ese entrecruzamiento específico se elabora la regulación de la vida social, distinción que coincide, al menos parcialmente, con la realizada por Durkheim entre los dos conceptos de integración y de regulación. El primero remite a la integración de los individuos a la sociedad, el segundo a la integración de la sociedad. Podríamos continuar diciendo que la integración a la sociedad está asegurada por los lazos sociales que los individuos construyen en el transcurso de su socialización, adhiriendo a las normas sociales vigentes, y que la regulación deriva del entrecruzamiento normativo de esos lazos sociales que permiten la integración de la sociedad en su conjunto. Es en el sentido de esta regulación social global que hablamos de configuración de vinculación; se trata, en cierta forma, de la trama de la sociedad. La función de una configuración de vinculación es producir una coherencia normativa global con el fin de permitir a los individuos y a los grupos constituir sociedad, más allá de sus diferencias y rivalidades. Para hacer sociedad, no es necesario, según Durkheim, que las representaciones colectivas estén presentes en cada conciencia individual, la pluralidad es una de las características fundamentales de las sociedades modernas. Pero es importante que ciertas representaciones sean compartidas por la mayoría, si no por todos. Es también en ese sentido que se puede hablar de una economía moral de los lazos sociales.

Definir el tipo de configuración de vinculación que caracteriza una sociedad determinada significa buscar, en las distintas capas de su historia y en las raíces antropológicas de su desarrollo, lo que conformó su trama específica. En otras palabras, el desafío consiste en pasar

3 En las sociedades totalitarias, donde el derecho a voto no existe y donde las libertades fundamentales no son reconocidas, no se puede hablar de lazo de ciudadanía propiamente tal. Se hablará más bien de un lazo que puede unir los individuos a su nación a pesar del régimen político de restricciones en que viven. 
de una tipología de los lazos sociales (en el sentido de la vinculación de los individuos a los grupos) a una tipología de las configuraciones de vinculación (en el sentido de la regulación normativa de los lazos sociales en las sociedades modernas). En cada régimen de vinculación los cuatro tipos de lazos sociales pueden tener una función de integración y/o una función de regulación. Un lazo integrador es un lazo que une al individuo con un grupo, mientras que un lazo regulador tiene una función suplementaria de adhesión; función que consiste en producir un conjunto de reglas y de normas susceptibles de expresar la extensión de su influencia a los otros lazos, hasta permear su concepción normativa inicial. Genera valores y principios de educación moral susceptibles de extenderse hacia el conjunto de la sociedad. Un lazo regulador es, en cierta forma, un lazo preeminente.

A partir de esta definición preliminar se pueden distinguir cuatro tipos de configuración-o de régimen- de vinculación: las configuraciones de tipo familiarista, voluntarista, organicista y universalista (ver cuadro siguiente).

\section{CUADRO 1}

Tipología de configuraciones de vinculación

\begin{tabular}{|l|c|c|}
\hline & $\begin{array}{c}\text { LAZOS } \\
\text { INTEGRADORES }\end{array}$ & $\begin{array}{c}\text { LAZO PREEMINENTE, } \\
\text { INTEGRADOR Y } \\
\text { REGULADOR }\end{array}$ \\
\hline Configuración de tipo familiarista & LPE/LPO/LC & LF \\
\hline Configuración de tipo voluntarista & LF/LPO/LC & LPE \\
\hline Configuración de tipo organicista & LF/LPE/LC & LC \\
\hline Configuración de tipo universalista & LF/LPE/LPO & \\
\hline
\end{tabular}

LF: lazo de filiación - LPE: lazo de participación electiva - LPO: lazo de participación orgánica - LC: lazo de ciudadanía

En cada configuración existe, en consecuencia, un lazo preeminente. En la configuración de tipo familiarista, es el lazo de filiación; en la configuración de tipo voluntarista, el lazo de participación electiva; en la configuración de tipo organicista, el lazo de participación orgánica; y, por último, en la configuración de tipo universalista, el lazo de ciudadanía.

Examinemos ahora los factores más frecuentemente asociados a estos cuatro tipos de configuración ${ }^{4}$ : el nivel de desarrollo económico, la relación con las desigualdades, el sistema de protección social y el civismo.

4 Al hablar de "factores asociados" y no de "factores explicativos", queremos subrayar que la causalidad no descansa en estos factores considerados separadamente; sino que estos pueden jugar conjuntamente. Son, la 
El nivel de desarrollo económico es a veces difícil de apreciar de manera satisfactoria, ya que los indicadores de referencia son a menudo discutibles. No entraremos en los infinitos debates filosóficos que estas cuestiones suscitan. Solo retendremos que una sociedad económicamente desarrollada, ya sea esta emergente o postindustrial, se define antes que todo por la organización del trabajo, por el uso de las ciencias y de las técnicas y por las consecuencias económicas y sociales de esa racionalización de la producción (Aron, 1988). Es evidente que existen brechas importantes entre los países y las regiones, inclusive al interior de la Unión Europea. También existen, al interior de cada país, regiones más rurales que otras y donde el artesanado y la pequeña empresa constituyen la base de la actividad económica.

La relación con las desigualdades es otro factor que puede asociarse a los regímenes de vinculación. La percepción de las desigualdades varía considerablemente de una sociedad a otra: en algunas, estas son consideradas como inevitables y no suscitan ningún tipo de indignación moral, mientras que en otras, por el contrario, son la expresión de una disfuncionalidad o de un malestar social que es necesario combatir con urgencia. La sensibilidad social a esta cuestión puede también variar de acuerdo con la coyuntura económica.

El sistema de protección social puede ser considerado a partir del criterio de "desmercantilización" (decommodification). Hacer de los individuos otra cosa que una mercancía intercambiable: así fue el desafío del Estado social al fin de la Segunda Guerra Mundial. Pero ese proceso de "desmercantilización" no fue llevado a cabo con igual intensidad en todos los países del mundo occidental y existen, por consiguiente, grandes variaciones que deben ser tomadas en cuenta, como lo demostró Gøsta Esping-Andersen (1990).

Finalmente, el civismo es una noción fundamental para medir el grado de compromiso de los individuos con el servicio a la colectividad, que constituyen por medio de su asociación. Robert Putnam la señaló como una dimensión del capital social. En ese sentido, el término remite tanto a una característica individual -los individuos están más o menos comprometidos en relaciones sociales- como a una exigencia moral del sistema social (Putnam, 2000).

\section{CONFIGURACIÓN DE TIPO FAMILIARISTA Y NATURALIZACIÓN DE LA POBREZA}

La configuración de tipo familiarista está regulada por la influencia que asegura el lazo de filiación en los otros tipos de lazo. Se encuentra más extendido en las regiones caracterizadas por un débil desarrollo industrial, en zonas rurales donde la economía está basada todavía en gran medida en pequeñas unidades de producción relativamente replegadas sobre sí mismas o sobre un sector geográfico limitado. Sin embargo, puede mantenerse en regiones más desarrolladas, ofreciendo así una base familiarista a un capitalismo de pequeños empresarios solidarios entre ellos. Puede también caracterizar el modo de desarrollo de un país emergente en el que las estructuras modernas de la economía se conjugan con la supervivencia de tradiciones marcadas por el solidarismo familiar. Este régimen se acompaña

mayor parte del tiempo, interdependientes, y es esa interdependencia que constituye, creemos, la clave de la explicación causal más probable. 
de enormes desigualdades sociales, sin que por ello estas sean decididamente combatidas. El sistema de protección social no es solamente incompleto, sino igualmente, la mayoría de las veces, de naturaleza clientelista. La aplicación del principio de "desmercantilización" (decommodification) es tan limitado que los individuos y los hogares más pobres no pueden acceder a condiciones de existencia realmente seguras frente a los avatares de la vida. Por último, el civismo es muy débil: el mercado de trabajo puede estar controlado por la mafia o por redes locales organizadas; el personal político está a menudo corrompido y las instituciones públicas suelen estar volcadas en beneficio de los intereses individuales o categoriales. La configuración de tipo familiarista promueve una muy importante solidaridad familiar para hacer frente a la pobreza, la que sigue siendo masiva en la medida que el mercado laboral suministra pocas protecciones generalizadas y permite que se desarrolle una economía informal al margen de la condición del salario mínimo.

Este tipo de configuración constituye en sí mismo el factor explicativo de eso que hemos Ilamado la pobreza integrada (Paugam, 2005); podemos ver ahí las condiciones sociológicas de la "naturalización" de la pobreza. Los pobres cumplen una función precisa en el sistema social y no están estigmatizados a título individual. Los fines domésticos no están subordinados a los fines nacionales y el Estado presenta dificultades para imponer una reflexión de un nivel superior que sea capaz de liberarse de la sola preservación de los intereses personales. En otras palabras, los sentimientos oscuros y los prejuicios que operan en la sociedad persisten a pesar de la influencia que pueden ejercer a nivel mundial las representaciones opuestas. Ahora bien, la "naturalización" de la pobreza forma parte de ellos. Esta última constituye una creencia colectiva que permite que cada sector de la sociedad conserve su lugar en el sistema de estratificación social sin que se tenga el sentimiento de que esto es injusto o el producto de una disfuncionalidad de la sociedad. Esta creencia es aún más notable cuando es compartida tanto por los ricos, que obtienen algunos privilegios de esta situación, como por los pobres, que se aseguran, por la aceptación de este destino, de una integración social mínima. No obstante, raramente la interiorización de esto es total y los pobres no están siempre enteramente convencidos de pertenecer a una categoría naturalmente inferior; no son pasivos y pueden manifestar capacidad de resistencia o de protesta, las investigaciones sociológicas lo han demostrado de manera reiterada 5 . En cambio, se constata en este tipo de configuración una cierta rutinización de la dominación, sobre todo en la medida en que está asociada a formas de protección paternalista, contribuyendo a mantener en el substrato social la idea de una naturalización de la pobreza.

En una configuración de tipo familiarista, tanto los ricos como los pobres tienen la convicción de que el fundamento principal de su integración y de su bienestar es la familia: es ella quien constituye el soporte privilegiado de su protección y de su reconocimiento ${ }^{6}$. Es posible destacar que esta representación de la pobreza como un destino está reforzada por el rol que juega la religión católica -que valora la pobreza-, especialmente en las regiones

5 Incluso en una relación de asistencia existen formas de resistencia al estigma y de negociación del estatus. Ver acerca de este punto: Paugam (2013c) y Duvoux (2009).

6 En algunos países la Constitución indica claramente que la familia es la célula básica de la sociedad. 
pobres donde la tasa de práctica religiosa regular es más elevada. En Europa, la particularidad de los países del sur en relación con los países del norte, es que en los primeros son claramente más numerosos los pobres que comparten de manera permanente esa condición. La pobreza puede ser más difusa y puede amortiguarse más fácilmente en el seno del grupo, en especial mediante la práctica religiosa: en una región pobre, esta última consagra en cierta medida una forma de pertenencia a la comunidad local de los pobres -recordemos que existe todavía en estas regiones rurales poco desarrollo y una ostensible religiosidad popular. Como la religión cristiana valora también a la familia, los pobres extraen de ella tanto la racionalización de su existencia como padres, como sus esfuerzos cotidianos para ocuparse de sus hijos, inclusive cuando estos se transforman en adultos. Ser pobre entre los pobres de estas regiones significa en definitiva ser pobres en familia y unidos por la misma práctica religiosa; en ese sentido sería posible decir que la pobreza está integrada social y religiosamente. No se trata aquí de concluir que el discurso de naturalización de la pobreza es mantenido y determinado por la religión católica en los países y regiones pobres ${ }^{7}$, sino de destacar que los pobres pueden encontrar en su práctica religiosa formas de compensación a sus dificultades cotidianas y los ricos una confirmación de su dominación "natural", que una caridad cotidiana hacia los miserables puede tornar socialmente aceptable.

Esta representación de la pobreza está todavía muy extendida en la India, donde las solidaridades familiares son potentes. Sin embargo, estas últimas son en alguna medida integradas a lógicas de asignación estatutaria que encontramos en el sistema de castas, incluso si esta forma de discriminación está oficialmente prohibida. En la investigación cualitativa acerca de la percepción de la pobreza realizada recientemente por Jules Naudet en los barrios ricos de Delhi ${ }^{8}$, la naturalización de la pobreza opera en su inicio bajo un registro religioso. Según la teoría del karma, los pobres nacen pobres en razón de sus actos en vidas precedentes. Sin embargo, las entrevistas recogidas confirman también, más allá de esta dimensión religiosa, la creencia notablemente expandida de que los grupos sociales, en virtud de su cultura de casta y de clase, de su origen regional, no pueden mezclarse. Los pobres estarían entonces naturalmente predispuestos a determinados modos de vida ${ }^{9}$. Observamos que, en su forma extrema, la naturalización de la pobreza puede incluso conducir a su negación: aquellos que han sido calificados de "pobres" lo son en relación con otras categorías sociales, no lo son si se considera que pertenecen a otro mundo,

7 Existen, ciertamente, excepciones. Por ejemplo, la referencia a la teología de la liberación en América Latina ha tenido por efecto estructurar las luchas campesinas o populares en numerosas regiones pobres en nombre de una lectura específica del evangelio no reconocida -o reconocida solamente de manera parcial y minoritariapor la Iglesia católica.

8 Se trata de un programa ANR (Agence Nationale de la Recherche-Agencia Nacional de la Investigación) titulado: "La elite y los pobres", el que se apoya en una investigación comparativa realizada en los barrios altos de París, São Paulo y Nueva Delhi. El equipo se compone de Serge Paugam como coordinador, Bruno Cousin, Camila Giorgetti y Jules Naudet como los encargados respectivos de los tres terrenos.

9 Respecto de la teoría del karma nos referiremos particularmente a Max Weber y a su trabajo: Hindouisme et bouddhisme (Flammarion, col. Champs, París, 2003). Jules Naudet presenta esta teoría, siguiendo a Louis Dumont, confrontándola con otras formas de justificación del fenómeno jerárquico (Postface: les sociodicées ou la justification des privilèges, en C. Jaffrelot y J. Naudet (2014): Justifier l'ordre social, PUF, col. La vie des idées, París, pp. 77-93). 
cuyos criterios de organización familiar y de integración social son específicos. Si bien la naturalización de la pobreza sigue muy vigente en India, la mayoría de los investigadores advierten hoy que los "intocables" no están completamente convencidos de su impureza y que existe igualmente en ellos una distancia crítica al estigma. No obstante, a pesar de que este proceso pueda ser o no enteramente interiorizado por los pobres mismos, el hecho es que perdura. Es preciso entonces atribuir dicho fenómeno al sistema de castas y, más aún, a una configuración de tipo familiarista, siempre que subrayemos la particularidad india de la implicación estatutaria, rígida y persistente del lazo de filiación. En este caso, la vinculación a la familia está, de forma inevitable, ligada a la vinculación a una comunidad etnorracial o a un grupo de casta.

Encontramos también este proceso de naturalización de la pobreza en países como Brasil, donde las desigualdades son igualmente fuertes y la solidaridad familiar importante. Esto está presente particularmente en los discursos de los habitantes de los barrios altos. Estos últimos ven en los pobres una población tan al margen de la sociedad, tan por fuera de la civilización, que es necesario ya sea protegerse de ellos por medio de un sistema refinado de seguridad (marcada delimitación del espacio privado, videovigilancia, guardias permanentes en las entradas de los edificios...), ya sea reeducarlos completamente, lo que se expresa en el momento en que se trata de hacer de ellos empleados domésticos (Giorgetti, 2015). Una relación de dominación tradicional, en parte heredada de la época de la esclavitud (muy tardíamente abolida en este país), teñida eventualmente de paternalismo benevolente, subyace así en esta representación de inferioridad natural de los pobres. No obstante, estos últimos han podido emprender luchas para intentar mejorar su condición -el Movimiento de los Sin Tierra es una de las expresiones más emblemáticas de estos últimos años-. A su vez, el programa nacional de lucha contra la pobreza ("Bolsa familia"10), así como las leyes sociales que enmarcan cada vez más el mundo del trabajo, imponen progresivamente otra representación de la pobreza y los mismos pobres toman conciencia de sus derechos, lo que se traduce en algunos casos en relaciones más conflictivas con las categorías superiores. Dicho de otra manera, es cuando los pobres comienzan a desprenderse de la influencia que ejerce en ellos su entorno familiar-sobre todo cuando ella es exclusiva- y de la relación de dominación que los une a los ricos, que logran juzgar su condición inferior como no natural, a planear para ellos y sus familias otro destino y a imponer así otro sistema de representación de la pobreza. Este proceso de "desnaturalización" de la pobreza es intensificado cuando se saben protegidos por un Estado que les asegura no solamente los derechos civiles y políticos, sino también los derechos sociales.

Los ejemplos que acabamos de plantear confirman que existe en el tipo de configuración familiarista un terreno favorable al proceso de naturalización de la pobreza. Dos puntos deben ser precisados. En primer lugar, no es el hecho de estar estrechamente apegado a su familia lo que refuerza la naturalización de la pobreza, sino el hecho de vivir en una sociedad donde la cohesión descansa en la preeminencia del lazo de filiación de los otros tipos de

10 El título de este programa ("Bolsa de familia") es en sí mismo revelador del concepto de protección social. La ayuda aportada a la familia -y no al individuo- está condicionada a la obligación de escolarizar a los niños. 
lazo. En este tipo de configuración, la familia es la célula básica de la sociedad, y todos los estratos sociales, tanto los pobres como los ricos, deben ajustarse a ella porque es ahí donde pueden encontrar los recursos que necesitan. Si la pobreza debe ser combatida -algo que no genera consenso- es ante todo por el esfuerzo de cada uno en la relación que teje con los miembros de su familia, ya sea esta acomodada o desfavorecida. Mientras la moral doméstica esté llamada a regular de manera prioritaria la vida social en general, los intereses personales a menudo prevalecen sobre los intereses colectivos, la tolerancia respecto de las desigualdades encuentra pocos límites y la naturalización de la pobreza constituye un poderoso modo de racionalización. En segundo lugar, esto no quiere decir que este proceso de naturalización no pueda aparecer en los otros tipos de configuración de vinculación. Las formas más o menos radicalizadas de racismo están presentes en todas las sociedades y se fundan en el principio de inferioridad "natural" de algunas categorías de la población. La perversidad del racismo consiste en permitir que, sin renegar de los ideales fundadores de su comunidad política, ciertos estratos de la sociedad justifiquen su deseo común de excluir a los grupos minoritarios -los que son también los que con mayor frecuencia se encuentran en situación de pobreza-, bajo pretexto de que ellos serían "naturalmente" diferentes. Planteamos la hipótesis de que este proceso de naturalización de los pobres estará menos desarrollado en los otros tipos de configuración y que, cuando sea visible, será bajo una forma diferente, asociándose a otras representaciones de la pobreza.

\section{CONFIGURACIÓN DE TIPO VOLUNTARISTA Y CULPABILIZACIÓN DE LOS POBRES}

La configuración de tipo voluntarista es regulada, como vimos, por el lazo de participación electiva. Este lazo está estrechamente ligado al principio de que nada debe obstaculizar la libertad de asociación a base del principio de afinidad electiva, sea este último motivado o no por la búsqueda del interés individual. Esta configuración comporta, por consiguiente, una gran correlación con el principio de la libertad de empresa. Dicho principio es compartido por la gran mayoría de la población, que acepta tanto más fácilmente las reglas del mercado en cuanto estas se conforman al deseo, juzgado legítimo, de enriquecimiento personal. Es lógico, por tanto, que esta configuración ofrezca todas las condiciones necesarias al desarrollo del capitalismo. Pero si el liberalismo -y por esta razón, el mercado- encuentra un terreno favorable en la configuración de tipo voluntarista, no constituye por ello una dimensión exclusiva. El mercado está presente en todos los regímenes, incluso en el régimen familiarista. En la actualidad, el crecimiento de los países emergentes se ve, de alguna manera, posibilitado socialmente por esa configuración de tipo familiarista.

En el régimen voluntarista, las desigualdades son enormes y generalmente legitimadas por el mérito de los más voluntariosos y los más audaces. El sistema de protección social descansa en gran medida en el cálculo libre de la gestión de riesgos por medio de aseguradoras privadas. Se presenta principalmente bajo la forma de una asistencia mínima focalizada hacia los sectores más pobres de la población. En esta configuración, el civismo es en cambio particularmente dominante, al igual que la vida asociativa es floreciente, y las fundaciones privadas orientadas a defender los intereses de la sociedad civil, muy dinámicas. Está anclado prioritariamente en 
un sistema de pertenencia comunitaria inspirado por aspiraciones colectivas de ciudadanía. La configuración de tipo voluntarista asocia, en definitiva, un nivel de protección social débil y una incitación a la participación solidaria en agrupaciones por afinidad.

La pobreza es a menudo percibida como un efecto social inevitable, ya que supone que se debe castigar a los menos capaces y/o los menos osados. En estas condiciones, los más pobres no tienen otra salida que la de contar consigo mismos para escapar de su condición, lo que se asocia con el mito del self-made man. Por lo demás, cuando para sobrevivir dependen de mecanismos institucionales de asistencia, son frecuentemente juzgados como irresponsables, tramposos o perezosos. La culpabilización de los pobres es el reflejo de la importancia que se le da a la noción de mérito. Los pobres no son naturalmente pobres, como en la configuración anterior, ya que se espera que ellos no estén cómodos en su condición; sin embargo la ideología del voluntarismo y del mérito no alcanza a sacudirse completamente de la creencia compartida de que la pobreza puede implicar también desigualdades de talentos y por consecuencia, cualidades consideradas como "innatas"11. La ambigüedad de los discursos fundados en el mérito es que mezclan muy frecuentemente argumentos que se apoyan en características tanto innatas como adquiridas.

Varios eurobarómetros dedicados a la percepción de la pobreza han sido realizados desde mediados de los años 1970 (Paugam y Selz, 2005). Dentro del conjunto de preguntas realizadas en esas encuestas, una hacía referencia a las causas de la pobreza. Las respuestas permitían distinguir dos explicaciones tradicionales y radicalmente opuestas, una que resaltaba la pereza o la mala voluntad de los pobres, y otra que subrayaba, por el contrario, la injusticia imperante en la sociedad. Es sorprendente constatar que el Reino Unido aparece, cualquiera sea la fecha de la encuesta, como el país donde la proporción de personas que explican la pobreza por la pereza es la más elevada. La brecha entre el Reino Unido y Francia era particularmente alta en 1976, ya que más de 44\% de ingleses pensaban así, frente a alrededor de $17 \%$ de franceses. En 2009, después de cuarenta años de crisis, la brecha persistía de manera sustancial: aproximadamente uno de cada cuatro ingleses daba aún esa explicación, frente a menos de uno de cada diez franceses. Existen, en esos dos países, explicaciones diferentes de la pobreza, como lo han demostrado otros trabajos (Evans, Paugam y Prélis, 1995). Ambos son países centralizados, y el modo de regulación de los problemas sociales se ha prestado para debates de envergadura nacional. La cuestión social es formulada de maneras distintas. La visión francesa se inscribe sin lugar a dudas en la tradición durkheimiana de la solidaridad orgánica, considerada hoy amenazada por el incremento de la "exclusión", mientras que la visión británica está fundada en una concepción de la sociedad en la que los individuos deben poder disponer de recursos suficientes no para evitar la marginalización, sino para afrontar la competencia con otros en un mercado abierto donde las diferencias estatutarias son débiles e incluso inexistentes. En el primer caso, la sociedad preexiste a los individuos y debe regularse para permitir el bienestar de cada uno y la cohesión del conjunto social; en el segundo, son los individuos que, aceptando la lógica del mercado y

11 Varios trabajos han sido consagrados al tema de la meritocracia: Young (1958) y McNamee y Miller (2004). 
de la competencia, deben hacerse cargo por sí mismos de su propia protección, incluso si se concuerda en que deben ser ayudados de manera mínima en caso de necesidad.

La culpabilización de los pobres también es muy habitual en Estados Unidos. El ascetismo protestante impuso las representaciones del éxito y de la pobreza, y estas continúan siendo estructurantes. En los años 1960, el momento en que se redescubrió la pobreza en el país (Harrington, 1967), esta era percibida como una anomalía, como la otra cara del progreso en una sociedad rica. La "guerra incondicional contra la pobreza", declarada por el presidente Johnson en 1964, incluía numerosas reformas al sistema de ayudas, pero, como lo subrayó Robert Castel, la intervención social conservó siempre en ese país una naturaleza psicologizante. Los pobres eran, ante todo, considerados como personas que presentaban signos de discapacidad o de carencias individuales (Castel, 1978). En los años 1980, con la llegada de Reagan a la presidencia, el discurso de denuncia respecto de la "pobreza voluntaria" encontró notorias resonancias entre los universitarios conservadores, particularmente en Charles Murray (1984) -para quien la AFDC (Aid to Families with Dependent Children) tenía un efecto de desincentivo al trabajo- y en Lawrence M. Mead (1986). Es en ese espíritu que se desarrollaron entonces los programas de workfare (Katz, 1986, 1989), los que lejos de representar únicamente el ala tradicional de la sociedad estadounidense, fueron llevados adelante, en los años 1990, de manera consensual tanto por los liberales (es decir, los progresistas, en el sentido norteamericano) como por los conservadores (Morel, 1996: Duvoux, 2014). Ciertamente, Estados Unidos y el Reino Unido no son los únicos países que se orientaron hacia ese tipo de programas y a desarrollar ese tipo de juicios acerca de los pobres dependientes de la asistencia social: Francia, como se sabe, se ha inspirado recientemente de este tipo de políticas poniendo en práctica el RSA (Ingreso de Solidaridad Activa) ${ }^{12}$, cuyo objetivo es justamente incitar a los pobres a aceptar los pequeños trabajos como complemento de sus ingresos mínimos (Paugam y Duvoux, 2008; Duvoux, 2012), y Alemania también ha reformado su sistema de protección social en un sentido similar (Boget, 2014). Existe sin embargo en Estados Unidos y en el Reino Unido una propensión más alta a llegar a un consenso social y político respecto de este tipo de programas. Debemos entender, sin duda, que se trata del efecto de la configuración de vinculación de tipo voluntarista, de la que estos dos países son particularmente cercanos.

\section{CONFIGURACIÓN DE TIPO ORGANICISTA Y VICTIMIZACIÓN CONTROLADA DE LOS POBRES}

La configuración de tipo organicista está dominada por el lazo de participación orgánica, que gobierna por su fuerza reguladora todos los demás $\operatorname{lazos}^{13}$. Derivada de la terminología

12 En francés: Revenu de solidarité active.

13 Al calificar de "organicista" este tipo de configuración, no nos estamos inscribiendo en la perspectiva teórica del organicismo, que fue una corriente bastante cuestionada en ciencias sociales. No se trata de decir que todas las sociedades pueden ser analizadas como organismos vivos; al contrario, la tipología de las configuraciones de vinculación está fundada en el reconocimiento de la diversidad de las sociedades. Utilizamos esta expresión para calificar de manera metafórica e ideal-típica un modo particular de organización de la sociedad, inscribiéndonos 
durkheimiana, es posible comprenderla como la realización de la sociedad industrial, la solidaridad orgánica entendida como la expresión de la sociedad moderna fundada en la diferenciación de los individuos y la complementariedad de sus funciones. La configuración de tipo organicista está, en consecuencia, lógicamente ligada al desarrollo económico, a la intensificación de los intercambios en el mundo del trabajo y en la sociedad mercantil. Pero no corresponde únicamente a una fase avanzada de desarrollo histórico de las sociedades modernas -si fuera así, el tipo organicista podría caracterizar en último caso a todas las sociedades desarrolladas-. Este tipo particular remite también, y sobre todo, a una relación particular entre los individuos y el Estado, y a una sociedad donde la vinculación social descansa principalmente en una lógica de protección estatutaria, en gran medida orquestada por el poder regulador del Estado. En esta configuración, la participación en los intercambios pasa por la vinculación casi obligatoria a un cuerpo intermedio (en el sentido de una corporación profesional) que procura un estatuto considerado como garantía frente a los avatares de la vida. Cada grupo así constituido construye de esta manera un lazo con los demás y con el Estado, permitiendo relaciones de interdependencia asentadas en el principio de complementariedad. Este tipo de configuración implica que el Estado sea capaz de crear y mantener corporaciones en sectores estratégicos -se hablará de "corporativismo de Estado" -, y también de regular los demás sectores como órganos diferenciados susceptibles de asegurar el buen funcionamiento de la economía y la sociedad.

En esta configuración, si bien los distintos grupos constituyen órganos Ilamados a cooperar, pueden también llegar a rivalizar los unos con los otros. Con esto, las desigualdades aparecen como constitutivas de la vida social, no en el sentido de su naturalización, como en la configuración anterior, sino en el sentido de las luchas por la clasificación en la escala de prestigio y de las ventajas materiales que procura la dominación. En definitiva, en el régimen organicista el Estado interviene de manera claramente más visible que en los regímenes familiarista y voluntarista. Se dedica a asegurar su rol de regulación de los cuerpos profesionales y de organización de la negociación colectiva. La tolerancia respecto de las desigualdades disminuye, aun cuando subsisten formas de racionalización de las diferencias estatutarias a base de su carácter funcional. En realidad, los grupos que componen el cuerpo social son inevitablemente a la vez complementarios y rivales, a tal punto que se hace necesario un trabajo de coordinación y de pacificación de las luchas por parte del Estado.

En este tipo de configuración, el sistema de protección está más avanzado en el sentido de la "desmercantilización", pero sigue estando fragmentado en un sinfín de subsistemas diferenciados, expresando así una lógica de distinción estatutaria y de reivindicación categorial por la conquista de derechos específicos y por la defensa de beneficios adquiridos. En este régimen, el Estado ejerce una función clasificadora: jerarquiza tanto los estatutos de sus funcionarios como los de los grupos socioprofesionales que provienen de la sociedad civil (Schnapper, 1989). Finalmente, su acción procede por focalización de las categorías susceptibles

de esta forma en la continuación del pensamiento de Durkheim, que era también muy crítico de las teorías organicistas. 
de ser objeto de políticas específicas. El civismo está globalmente menos desarrollado que en la configuración de tipo voluntarista, ya que el interés general es postergado frente a los intereses particulares de los grupos rivales, los que esperan del Estado un arbitraje que ellos mismos, con frecuencia, son incapaces de asegurar por sí solos.

En una configuración de tipo organicista, los pobres no están fuera del sistema social, aunque sí constituyan de alguna forma el último estrato de la sociedad. Son a menudo percibidos como víctimas, frente a las cuales hay que intervenir de manera intensiva. En este tipo de configuración, todas las categorías, por muy diferentes que sean, tienen derecho a un estatuto social aceptable a la luz del principio de igualdad ciudadana, pero eso no implica necesariamente una igualdad de condiciones de vida. Es la razón por la que el mérito sigue siendo movilizado para justificar las diferencias socioeconómicas. La referencia a esta noción no tiene, sin embargo, la misma implicancia ideológica que en la configuración de tipo voluntarista: no se trata de acusar al conjunto de pobres de pereza -ya que estos últimos pueden haber experimentado circunstancias de vida muy severas que funcionan como atenuantes-, sino de valorar aquellas y aquellos que logran salir adelante por su trabajo, atribuyéndoles títulos privilegiados de distinción estatutaria, una suerte de victimización controlada de los pobres. Dicho de otro modo, en el nombre de los valores republicanos se valora tanto la solidaridad con las capas más desfavorecidas de la sociedad como el mérito que consagra a las élites dirigentes -es en este último sentido que se habla de "elitismo republicano" para calificar la inspiración ideológica del sistema escolar francés (Baudelot y Establet, 2009)-. Es interesante constatar que las categorías superiores entrevistadas en París, a diferencia de las interrogadas en São Paulo o Delhi, tienen un discurso muchas veces muy matizado acerca de los pobres, del que pueden surgir tanto justificaciones del "solidarismo" y del Estado social como garantía de la cohesión social mínima, como aquellas de las desigualdades estatutarias necesarias a la organización de la sociedad. En ambos casos, es efectivamente en nombre de una idea de pertenencia a un todo social compuesto de partes diferentes y complementarias, que se evita la culpabilización excesiva de los pobres -lo que no quiere decir que esa tendencia esté completamente ausente en las representaciones sociales-, al mismo tiempo que se evita toda idea de justicia social conducente a la nivelación social o al igualitarismo absoluto. La victimización controlada de los pobres conduce, en ese sentido, a conservar, generalmente sin formularlo de manera explícita, un sistema de tolerancia a las desigualdades estatutarias, sobre todo cuando estas son percibidas como una condición de la regulación económica y social, fundada en la interdependencia de las funciones y de los individuos.

La crisis de la sociedad salarial (Castel, 1995), cuyos signos más visibles son la precariedad profesional y el desempleo masivo, pone en cuestión, al menos parcialmente, el modo de regulación característico de la configuración de tipo organicista. Cuando los individuos han interiorizado las normas que sostienen el modelo de integración fundado en la preeminencia del lazo de participación orgánica, y siguen viviendo, a pesar de sus esfuerzos, en condiciones precarias, pueden experimentar un sentimiento de frustración y, en algunos casos, de inutilidad social. 


\section{CONFIGURACIÓN DE TIPO UNIVERSALISTA Y VICTIMIZACIÓN CONSOLIDADA DE LOS POBRES}

La configuración de tipo universalista está regulada antes que todo por el lazo de ciudadanía. Este implica una gran capacidad para hacer efectivo el principio democrático de la igualdad de los individuos, no solamente en relación con los derechos, sino también de manera más general en el funcionamiento de la vida económica y social. Este régimen es compatible con un alto nivel de desarrollo económico. Las reglas del mercado son aceptadas y reúnen, en muchos aspectos, mayor consenso que en la configuración de tipo organicista. La cuestión no es la de rechazarlas, ni de esquivarlas, sino de socializarlas. Para lograrlo, numerosos diálogos son organizados entre los actores sociales, que consiguen así superar sus intereses categoriales para hacer prevalecer el interés general y los valores de pertenencia a una comunidad de ciudadanos.

En un régimen universalista, el Estado es asunto de todos, se confunde con la noción misma de sociedad. Evitar las desigualdades extremas y la pobreza es también la expresión de un acuerdo casi general de convivencia, sin que nadie manifieste de manera ostentosa la superioridad eventual de tal o tal estatus. La idea de una subordinación impuesta y asfixiante es contraria a los principios de la vida social ordinaria; nada debe venir a entorpecer la voluntad de la emancipación individual, bajo la condición obviamente de respetar las instituciones que los representan. El sistema de protección social es aquí muy avanzado: financiado tanto por el impuesto como por las protecciones sociales, permite alcanzar una "desmercantilización" de un nivel muy elevado. Finalmente, el civismo es -en este régimentambién muy poderoso. El asociacionismo se puede desarrollar de manera igual o mayor que en la configuración de tipo voluntarista, sin embargo aquí toma otro sentido, ya que el compromiso cívico pasa ante todo por el respeto a las instituciones públicas. Este tipo de configuración conjuga, finalmente, una condición salarial marcadamente protectora y una concepción de la ciudadanía y de los derechos individuales que hace posible la autonomía en relación con las formas primarias de solidaridad.

Incluso si es necesario evitar considerar a los nórdicos como un conjunto perfectamente homogéneo ${ }^{14}$, estos tienen en común la cercanía con esta configuración de vinculación de tipo universalista. La victimización de la pobreza está más consolidada en estos países, ya que es en sí misma la expresión de la incapacidad de la sociedad para satisfacer su ideal de igualdad entre ciudadanos. En estos países, la industrialización fue tardía, sin embargo se logró rápidamente un consenso para proveer a todas las capas sociales -tanto campesinas como obreras- de un sistema común de protección social cuya eficacia para reducir de manera preventiva la pobreza ha sido muchas veces demostrada. Esta búsqueda de consenso está presente tanto en el mundo del trabajo como en la vida asociativa en general ${ }^{15}$; nada se

14 Ver acerca de este punto el libro de Yohann Aucante, Démocraties scandinaves (Armand Colin, París, 2013).

15 En un artículo reciente, Lars Tragardh examina desde un punto de vista teórico la conjunción en los países nórdicos de un alto nivel de intervención del Estado y una movilización importante de la población por medio de la vida colectiva. Ver: Rethinking the Nordic welfare state through a neo-hegelian theory of state and civil society, Journal of Political Ideologies, 15 (3), 2010, pp. 227-239. 
opone a las funciones que cumple el Estado en cuanto a la regulación de la sociedad civil, porque esta última está, ella misma, llamada a participar de esta regulación.

Una de las características de la realidad de la vida en sociedad en esta configuración es que la pobreza es considerablemente menos visible que en los otros países. En los eurobarómetros acerca de la pobreza es impactante constatar que Dinamarca es siempre el país de la Unión Europea donde las personas entrevistadas son proporcionalmente las que menos declaran percibir la pobreza -y mucho menos la extrema pobreza- alrededor de ellas. Preguntarles por las causas de la pobreza es incluso problemático, ya que este fenómeno les resulta en gran parte extraño. Esto no significa que no exista, pero es entendida como una cuestión marginal a cargo, sobre todo, de servicios especializados de acción social, los que se preocupan de erradicarla interviniendo específicamente, y de manera muy intensa, en los niños. Sin embargo, en la victimización consolidada de los pobres no deberíamos pensar que existe una ausencia total de violencia simbólica en las relaciones sociales entre los receptores de las ayudas sociales y los profesionales que prestan estos servicios. Los países nórdicos se vinculan a una ética del trabajo y ejercen presión hacia los pobres sin actividad. Esto, con el fin de que se reintegren rápidamente al mercado laboral; en tanto minoría, los pobres son objeto de un control social que se acompaña casi inevitablemente de una dura estigmatización. Ivar Lødemel ha estudiado de manera muy profunda el sistema noruego de asistencia social, comparándolo con el sistema inglés (Lødemel, 1997). En este contexto aparece una paradoja: mientras que este país, al igual que otros países nórdicos, está dotado de un sistema de protección social fundado en el principio considerablemente consolidado de la universalidad de derechos, la esfera de la asistencia no está por ello completamente libre de prácticas humillantes de las que es objeto una franja residual de la población que se beneficia de ella. Porque los pobres juzgados "meritorios" -particularmente las personas mayores y los discapacitados- han sido de cierta manera absorbidos por el sistema de seguridad, los trabajadores sociales intervienen entonces con los otros pobres, teniendo ante ellos el poder de decidir si se les otorgan o no las ayudas que solicitan. En esta perspectiva los pobres corren el gran riesgo de ser considerados sospechosos de no merecer las ayudas. Entonces son tratados como personas cuyo derecho a la asistencia no es automático. Esta dualización en el trato, que corresponde a una operación de filtraje de los pobres, ha contribuido a acrecentar el estigma de la asistencia. No deberíamos, sin embargo, hacer del sistema noruego de asistencia el ejemplo tipo de los países nórdicos. Por el contrario, el análisis detallado de estos sistemas nos conduce a poner el acento en las diferencias que existen entre ellos. Por ejemplo, el nivel de ayudas está definido de forma estandarizada e inscrito en la ley nacional en Finlandia y Dinamarca, mientras que está establecido localmente en Suecia y Noruega; implicando una determinación local definida por recomendaciones nacionales en Suecia, y dependiendo completamente de la decisión municipal en Noruega. Podemos entonces observar grandes variaciones regionales en Suecia y más aún en Noruega. Es interesante notar que el acceso a las ayudas supone criterios de elegibilidad variables, frecuentemente sujetos a la evaluación de los trabajadores sociales en Noruega, pero igualmente, aunque en menor medida, en Suecia. A pesar de estas diferencias, la paradoja estudiada por Ivar Lødemel sirve, me parece, para el conjunto de los países nórdicos. Existe un alto riesgo de estigmatización de los asistidos en la medida 
en que estos son considerados como el complemento residual de un tratamiento global y preventivo de la pobreza, que consiste en la inscripción de la gran mayoría de asistidos en la esfera deliberadamente más amplia de la seguridad social. En un sistema que apunta a la universalidad de los derechos, los asistidos no pueden sino ser percibidos como la expresión de un disfuncionamiento. Ellos reflejan por sí mismos el umbral -sin duda infranqueable- de la erradicación de la pobreza. Sin embargo, el proceso de victimización consolidado de los pobres es lo que legitima en estos países un tratamiento lo más global y preventivo posible de la pobreza. Esta es, en sí mismo, la expresión de una voluntad política y del consenso en torno a no permitir que ningún ciudadano, en razón de su condición económica y social, pueda quedar permanentemente fuera de la sociedad.

\section{CONSIDERACIONES FINALES}

No quisiéramos dar a entender aquí que la naturalización, la culpabilización y la victimización de la pobreza se explican nada más que por la trama de la sociedad o, como lo hemos visto, por la configuración específica de los lazos que vinculan a los individuos entre sí y con la sociedad misma. Estas formas de percepción de la pobreza son difusas y se encuentran inevitablemente en todas las sociedades. Podríamos incluso encontrarlas sucesivamente en el mismo individuo según los momentos específicos de su trayectoria y las fases de construcción de su personalidad. No obstante, hemos querido buscar -más allá de los factores de variación generalmente considerados en el análisis sociológico- lo relacionado específicamente con los modos de regulación de los lazos sociales, los que pueden ser estudiados a partir de una tipología de configuraciones de vinculación. De esta manera, este marco analítico constituye una clave de comprensión adicional y un instrumento eficaz para los estudios comparativos.

La naturalización de la pobreza puede ser analizada de esta manera como un factor asociado a una configuración de tipo familiarista, mientras que la culpabilización puede ser interpretada como una dimensión ligada a una configuración de tipo voluntarista. La victimización de los pobres remite, a su vez, tanto a la configuración de tipo organicista como a la configuración de tipo universalista, sin embargo, se encuentra más consolidada en el segundo tipo que en el primero. Dicho de otra forma, en la medida en que nos acercamos a una configuración de tipo universalista, la pobreza aparece cada vez más, en las representaciones colectivas, como una amenaza absoluta para la cohesión social. La pobreza no puede ser verdaderamente erradicada sino mediante políticas de prevención de riesgos, llevadas a cabo por la totalidad social y a costa de una voluntad compartida por todos los ciudadanos de vivir en una sociedad de semejantes, es decir, en una sociedad apacible, democrática y abierta a todos.

La tipología de las configuraciones de vinculación utilizada en este artículo resulta de una construcción ideal-tipo. Esta no tiene por función clasificar de manera estática a todas las sociedades sino, por el contrario, se esfuerza por determinar de manera dinámica los factores que las conducen a acercarse, en un momento dado de su historia, a uno u otro tipo. Este razonamiento teórico aumenta la posibilidad de interpretar el cambio. La relación que hemos 
establecido entre las configuraciones de vinculación y las formas de percepción social de la pobreza, no debe ser pensada como la marca inmutable de tal o tal sociedad: la posibilidad de constatar en el tiempo las transformaciones de estas percepciones de la pobreza, es en realidad tan alta como la posibilidad de ver a una sociedad pasar progresivamente de una configuración de vinculación a otra. En estas transformaciones de larga duración se mide toda la importancia del rol del Estado, no solamente porque protege y libera a los individuos, sino también porque produce un relato colectivo fundado en un conocimiento clarificador respecto de los mecanismos de integración de todos los ciudadanos.

\section{BIBLIOGRAFÍA}

Aron, R. (1988): Dix-huit leçons sur la société industrielle, Gallimard, reedición col. Folio Essais, París. Aucante, Y. (2013): Démocraties scandinaves, Armand Colin, París.

Baudelot, C. y R. Establet (2009): L'Élitisme républicain. L'école française à l'épreuve des comparaisons internationales, Seuil/La République des Idées, París.

Boget, Y. (2014): Le travail en dehors de l'emploi: la régulation du lien de participation organique et ses effets en Allemagne, en S. Paugam (dir.): L'Intégration inégale. Force, fragilité et rupture des liens sociaux, PUF, col. Le lien social, París, pp. 299-312.

Castel, R. (1978): La guerre à la pauvreté aux États-Unis: le statut de la misère dans une société d'abondance, Actes de la Recherche en Sciences Sociales, 19, pp. 47-60.

Castel, R. (1995): Les Métamorphoses de la question sociale. Chronique du salariat, Fayard, París.

Durkheim, E. (2007a): De la division du travail social, PUF, col. Quadrige, París.

(2007b): Le suicide. Étude de sociologie, PUF, col. Quadrige, París.

(2012): L'Éducation morale (1902-1903), PUF, col. Quadrige, París.

Duvoux, N. (2009): L'Autonomie des assistés. Sociologie des politiques d'insertion, PUF, col. Le lien social, París.

(2012): Le Nouvel Âge de la solidarité, Seuil/La République des Idées, París.

(2014): L'institutionnalisation de la précarité sur le marché du travail en France et aux Etats-Unis, en S. Paugam (dir.): L'Intégration inégale. Force, fragilité et rupture des liens sociaux, PUF, col. Le lien social, París, pp. 279-297.

Esping-Andersen, G. (1990): The three worlds of welfare capitalism, The Polity Press, Londres.

Evans, M., S. Paugam y J. Prélis (1995): Chunnel vision: Poverty, social exclusion and the debate on social welfare in France and Britain, Discussion Paper, Welfare State Programme/115.

Geremek B. (1987): La Potence ou la pitié. L'Europe et les pauvres du Moyen Âge à nos jours (1978), Gallimard, París.

Giorgetti, C. (2015): Comment les catégories supérieures de São Paulo parlent de leurs employées domestiques: analyse d'un rapport de classe, Brésil(s), Sciences Humaines et Sociales, 8, pp. 73-96.

Grannoveter, M. (1973): The strength of weak ties, American Journal of Sociology, 78 (4), pp. 1360-1380.

Harrington, M. (1967): L’Autre Amérique. La pauvreté aux Etats-Unis, Gallimard, París.

Katz, M.B. (1986): In the shadow of the poorhouse. A Social history of welfare in America, Basic Books, Nueva York. 
(1989): The undeserving poor. From the war on poverty to the war on welfare, Pantheon Books, Nueva York.

Lødemel, I. (1997): The welfare paradox. Income maintenance and personal social services in Norway and Britain, 1946-1966, Scandinavian University Press, Oslo.

McNamee, S.J. y R.K. Miller (2004): The meritocracy myth, Rowman and Littlefield, Lanham.

Mead, L.M. (1986): Beyond entitlement: The obligations of citizenship, Free Press, Nueva York.

Morel, S. (1996): Le workfare aux Etats-Unis, en S. Paugam (dir.) : L'Exclusion. L'état des savoirs, La Découverte, París, pp. 472-483.

Murray, C. (1984): Losing ground: American social policy, 1950-1980, Basic Books, Nueva York.

Naudet, J. (2014): Postface: les sociodicées ou la justification des privilèges, en C. Jaffrelot y J. Naudet: Justifier l'ordre social, PUF, col. La vie des idées, París, pp. 77-93.

Paugam, S. (2005): Les formes élémentaires de la pauvreté, PUF, coll. Le lien social, París.

(2013a): Les cycles de la solidarité envers les pauvres, en R. Castel y N. Duvoux (dir.): L’Avenir de la solidarité, PUF, col. La vie des idées, París, pp. 23-41.

(2013b): Le lien social, PUF, col. Que sais-je?, París.

(2013c): La disqualification sociale. Essai sur la nouvelle pauvreté, PUF, reedición col. Quadrige, París.

Paugam S. y M. Selz (2005): La perception de la pauvreté en Europe depuis le milieu des années 1970. Analyse des variations structurelles et conjoncturelles, Économie et Statistique, 383-384-385, 2005, pp. 283-305.

Paugam, S. y N. Duvoux (2008): La Régulation des pauvres, PUF, col. Quadrige, París.

Piven F.F. y R. Cloward (1993): Regulating the poor. The functions of public welfare, Vintage, Nueva York.

Putnam, R.D. (2000): Bowling alone. The collapse and revival of American community, Simon and Schuster, Nueva York.

Schnapper, D. (1989): Rapport à l'emploi, protection sociale et statuts sociaux, Revue Française de Sociologie, XXX-1, pp. 3-29.

Tragardh, L. (2010): Rethinking the Nordic welfare state through a neo-hegelian theory of state and civil society, Journal of Political Ideologies, 15 (3), pp. 227-239.

Van Oorschot W. y L. Halman (2000): Blame or fate, individual or social? An international comparison of popular explanations of poverty, European Societies, 2 (1), pp. 1-28.

Weber, M. (2003): Hindouisme et bouddhisme, Flammarion, col. Champs, París.

Young, M. (1958): The rise of meritocracy, Penguin Books, Londres.

Recibido: 11-9-2016

Aceptado: 29-11-2016 